\title{
Neue orale Antikoagulanzien stellen sich dem Vergleich
}

Fragestellung: Wie wirksam sind die neuen oralen Antikoagulanzien Dabigatran, Rivaroxaban oder Apixaban im Vergleich zu niedermolekularem Heparin wie Enoxaparin zur Prävention von tiefen Beinvenenthrombosen und Lungenembolien bei Patienten mit Hüft- und Kniegelenksersatz?

Hintergrund: Tiefe Beinvenenthrombosen und Lungenembolien sind schwerwiegende Komplikationen von Bettlägerigkeit und großen operativen Eingriffen. Diese thromboembolischen Komplikationen nahmen mit der Einführung der Thromboseprophylaxe mit Heparin rapide ab. In den letzten Jahren wurden sowohl in den Vereinigten Staaten als auch in Europa fast ausschließlich niedermolekulare Heparine zur Thromboseprophylaxe verwendet. Die neuen oralen Antikoagulanzien, die entweder wie Dabigatran direkte Thrombinhemmer oder wie Rivaroxaban und Apixaban Faktor-Xa-Hemmer sind, wurden alle zunächst in der Prävention tiefer Beinvenenthrombosen und Lungenembolien bei Patienten mit Hüft- und Kniegelenksersatz untersucht, bevor die großen Studien für Schlaganfallprävention bei Vorhofflimmern begonnen wurden. Die Autoren aus Spanien führten jetzt eine große Metaanalyse aller bisher durchgeführten randomisierten Studien mit den neuen oralen Antikoagulanzien durch.

Patienten und Methodik: Die Autoren führten zunächst eine Literaturrecherche durch um alle randomisierten Studien zu identifizieren bei denen die neuen Antikoagulanzien mit Enoxaparin bei Patienten mit Hüft- und Kniegelenksersatz verglichen wurden. Dabei wurden nur Studien berücksichtigt, bei denen die zugelassenen Dosierungen, nämlich 150 oder $220 \mathrm{mg}$ Dabigatran, $5 \mathrm{mg}$ Apixaban oder $10 \mathrm{mg}$ Rivaroxaban verwendet wurden. In den Studien die in Europa durchgeführt wurden, war die Vergleichstherapie Enoxaparin $40 \mathrm{mg} 1 \mathrm{x}$ täglich 12 Stunden vor dem operativen Eingriff und in den Vereinigten Staaten Enoxaparin $30 \mathrm{mg} 2 \mathrm{x}$ täglich 12 bis 24 Stunden vor dem operativen Eingriff. Die Endpunkte der Metaanalyse waren symptomatische tiefe Beinvenenthrombosen und symptomatische Lungenembolien. Der primäre Sicherheitsendpunkt waren klinisch relevante Blutungen.

Gómez-Outes A, TerleiraFernández Al, Suárez-Gea ML, Vargas-Castrillón E. Dabigatran, rivaroxaban, or apixaban versus enoxaparin for thromboprophylaxis after total hip or knee replacement: systematic review, meta-analysis, and indirect treatment comparisons. BMJ 2012; 344: e3675
Ergebnisse: Für die Metaanalyse wurden 16 randomisierte Studien verwendet mit insgesamt 38.747 Patienten. Dabei wurden jeweils vier Studien mit Dabigatran und Apixaban durchgeführt und acht Studien mit Rivaroxaban. Elf der 16 Studien benutzten die europäische Dosierung von Enoxaparin.
15 der 16 Studien waren doppelblind. Das mittlere Alter der Patienten lag zwischen 61 und 68 Jahren.

Die Ergebnisse für die einzelnen Antikoagulanzien werden im Folgenden alphabetisch vorgestellt: Für Apixaban ergab sich für den primären Endpunkt symptomatische tiefe Beinvenenthrombose und symptomatische Lungenembolie eine nicht signifikante Risikoreduktion von $18 \%$. Für Dabigatran ergab sich eine relative Risikominderung von $29 \%$, die ebenfalls nicht signifikant war. Rivaroxaban hatte eine statistisch signifikante 52 \%ige Risikoreduktion. Über alle drei Substanzen hinweg, ergab sich eine $35 \%$ ige Risikoreduktion, die statistisch signifikant war. In absoluten Zahlen traten 112 Ereignisse bei $19.481 \mathrm{~Pa}-$ tienten mit den neuen Antikoagulanzien auf und 133 von 16.668 bei Enoxaparin. Für Blutungskomplikationen ergab sich eine $18 \%$ ige Risikoreduktion zugunsten von Apixaban, die statistisch signifikant war. Für Dabigatran ergab sich eine $12 \%$ ige Risikoerhöhung, die statistisch nicht signifikant war. Für Rivaroxaban ergab sich eine $25 \%$ ige Risikoerhöhung, die statistisch signifikant war. Über alle Studien hinweg betrug das erhöhte Blutungsrisiko relativ gemessen $6 \%$. In Zahlen ausgedrückt, waren es 883 Ereignisse bei 19.481 Patienten unter den neuen Antikoagulanzien und 695 von 16.668 bei Enoxaparin. Berechnete man die Number needed to treat und die Number needed to harm pro 1.000 operierte Patienten, ergaben sich nur minimale Unterschiede zwischen den drei neuen Antikoagulanzien.

Schlussfolgerungen: Alle drei neuen oralen Antikoagulanzien sind im Vergleich zu Enoxaparin gut wirksam und haben zusammen genommen eine vergleichbare Rate der Blutungskomplikationen. Das etwas wirksamere Rivaroxaban führt auch etwas häufiger zu Blutungskomplikationen.

\section{- Kommentar von Prof. Hans-Christoph Diener Kaum Unterschiede}

Diese große Metaanalyse zeigt, dass es nur minimale Unterschiede zwischen den einzelnen Antikoagulanzien bezüglich Wirksamkeit und Risiko bei großen Gelenkoperationen und bezogen auf den Endpunkt tiefe Beinvenenthrombose und Lungenembolien sowie Blutungskomplikationen gibt. Es zeigt sich eine Tendenz dahingehend, dass die wirksameren Substanzen bezüglich der Prävention von Thromboembolien auch das etwas höhere Blutungsrisiko haben. In Einzelfällen mögen die relativen Risikoreduktionen und Erhöhungen dramatisch klingen. In absoluten Zahlen sind sie so gering, dass praktisch keine Unterschiede zwischen den neuen oralen Antikoagulanzien und Enoxaparin bestehen. Die neuen Antikoagulanzien haben den großen Vorteil, dass sie nicht injiziert werden müssen, sondern oral eingenommen werden können. 